\title{
Challenges of Pediatric Multisystem Inflammatory Syndrome Associated with Covid-19 - A Series of Clinical Cases
}

Ninel Revenco ${ }^{1,2, *}$, Silvia Foca ${ }^{1,2}$, Ala Jivalcovschi ${ }^{2}$, Larisa Ziaev ${ }^{1,2}$, Tatiana Turea ${ }^{1,2}$, Dina Bujor ${ }^{1,2}$, Ana-Mihaela Balanuța ${ }^{1,2}$, Ludmila $^{2}$ Serbenco $^{1}$, Irina Vasilieva ${ }^{1,3}$, Maria Vasilieva ${ }^{1,3}$, Ilie Vasiliev ${ }^{3}$

${ }^{1}$ State University of Medicine and Pharmacy "Nicolae Testemitanu".

${ }^{2}$ PHMI Mother and Child Institute.

${ }^{3}$ World Academy of Medical Sciences, Netherlands, Republic of Moldova.

*Corresponding Author: Ninel Revenco MD, PhD, Head of Department of Pediatrics, State University of Medicine and Pharmacy, "Nicolae Testemitanu", Republic of Moldova.

With advisory support: Ilie Vasiliev MD, Professor at the World Academy of Medical Sciences. First Superior Executor Vice- President of the World Academy of Medical Sciences. WAMS Coronavirus Safety Committee.

Received date: October 12, 2020; Accepted date: October 29, 2020; Published date: November 02, 2020

Citation: N Revenco, S Foca1, A Jivalcovschi, L Ziaev, I Vasiliev, et al. (2020) Challenges of Pediatric Multisystem Inflammatory Syndrome Associated with Covid-19 - A Series of Clinical Cases. Biomedical Research and Clinical Reviews. 1(4); DOI: 10.31579/2692-9406/027

Copyright:@2020 Ninel Revenco, This is an open-access article distributed under the terms of the Creative Commons Attribution License, which permits unrestricted use, distribution, and reproduction in any medium, provided the original author and source are credited.

\section{Background}

According to the first SARS-CoV-2 pandemic data, signs and symptoms were less frequently reported among pediatric patients versus adults. Later in April, was described as the multisystem inflammatory syndrome in children (MIS-C) associated with COVID -19. The clinical presentation of MIS-C includes fever, simultaneous involvement of two or more organ systems, altered inflammation parameters and laboratory or epidemiological evidence of SARS-CoV-2 infection. MIS-C features some similar clinical aspects with Kawasaki disease, toxic shock syndrome, and secondary hemophagocytic lymph histiocytosis syndrome / macrophage activation syndrome. This review describes the clinical features of 7 cases of MIS-C treated in the Republic of Moldova.

Keywords: multisystem inflammatory syndrome; children; covid-19; kawasaki disease; clinical case

\section{Introduction}

In children, with coronavirus2 (SARS-CoV-2) is responsible for mild respiratory symptoms, versus severe forms reported in adults. An association between SARS-CoV-2 /COVID19 and late manifestations of vasculitis has recently been suspected, especially in asymptomatic in children, which may be due to post-viral immunological reactions. MIS$\mathrm{C}$ recently described in pediatrics, in temporary association with COVID19 is characterized by a persistent fever, markers of inflammation and evidence of single or multi-organ dysfunction, MODS, and may include: features suggestive to Kawasaki disease, toxic shock or Macrophage activation syndrome, and a sepsis-like condition. MIS-C is used by the WHO. It is also known as PMIS-TS (Pediatric MIS temporarily associated with SARS-CoV-2 infection) in Europe and PMIS temporarily associated with COVID19 in the UK. Clinical presentation can vary widely, case reports release significant gastrointestinal symptoms, heart injury, mild respiratory symptoms and variable incidence of rash, conjunctival injection and involvement of the oral mucosa.

\section{Purpose of the study}

Cases review for a comprehensive presentation of MIS-C syndrome and COVID 19 - related Kawasaki disease.

\section{Materials and Methods}

The study represents the prospective analysis of 7 clinical cases with MIS$\mathrm{C}$ temporarily associated with SARS-CoV-2 infection hospitalized between April 22 and September 3, 2020. The analyzed variables included anamnestic, epidemiological, demographic information, general clinical manifestations, the results of laboratory and confirming to SARS-CoV-2 infection, imaging investigations and the evolution of the disease in accordance with the implemented therapeutic decisions. The criteria for including patients in MIS-C were in line with the revised WHO definition.

\section{Results}

The mean age of the 7 patients diagnosed with MIS-C was $6.6 \pm 2.1$ years, with limits between 8 months and 15 years. The distribution of children according to gender belongs to a prevalence of the syndrome among females $71.42 \%$ (5 girls). All patients included in the study showed at least one evidence of SARS-CoV-2 infection, three (42.85\%) of whom tested positive by RT-PCR in the nasopharyngeal swab, while the other 4 (57.15\%) (Cases with 1, 2, 3 and 4) presented serological evidence of IgG and / or Ig M anti-SARS-CoV-2 in a positive titer. Only one patient out of the seven had no concomitant pathologies. Comorbidities included surgically corrected congenital heart defects and Down syndrome (case 1), beta-thalassemia (patient 7), complicated cirrhosis of the liver with chronic liver failure, portal hypertension and hypersplenism (patient 6), cytomegalovirus infection (patients with 3 and 7), with EBV (patients 4 and 7) and parvovirus B19 (patient 3). The patient with 5 had a history of frequent acute viral respiratory infections complicated by laryngotracheitis. 
Symptoms were dominated by fever and skin rash in $100 \%$ of cases, abdominal pain and tachypnea (85.71\%), oral mucosal lesions, headache, myalgia and fatigue $(71.42 \%)$, non-purulent conjunctivitis, nonsuppurated lymphadenopathy and diarrhea in $57.14 \%$ of cases. A smaller number of patients presented cough $(28.57 \%)$, nausea / vomiting was manifest in about $14.28 \%$ of children. Three of the 7 patients $(42.85 \%)$ developed MIS-C similar to Kawasaki disease, meeting the criteria of the American Society of Cardiology for Kawasaki Disease. At admission, they had a persistent, drug-resistant fever lasting more than 5 days, unilateral non-suppurated lymphadenopathy, bilateral non-purulent bulbar conjunctivitis, polymorphic skin rash without blisters or crusts ,oral mucosal changes like red, cracked lips, raspberry tongue with hypertrophied papillae and diffuse oropharyngeal erythema, as well as skin changes expressed by palmar and plantar erythema in the initial stage.

Echocardiographic examinations performed no signs of impaired myocardial contractility, pericardial effusion or aneurysmal dilation of the coronary arteries in any of the 6 patients. Although respiratory symptoms were poor in most patients, chest radiography showed uni- or bilateral pneumonia in $71.42 \%$ of patients, in 2 of them complicated by pleural effusion.

In the early stages of the disease, most patients presented with neutrophilic leukocytosis and lymphopenia, which returned to normal underlining the treatment. Thus, leukocytosis was present in 4/7 (57.14\%) cases, registering a maximum value of $38.2 \times 109$ / L, the ESR was increased to $5 / 7(71.42 \%)$. ) of cases, the mean value of which was 30.28 $\pm 6 \mathrm{~mm} / \mathrm{h}, 57.4$ percent had hyperfibrinogenemia, and $28.57 \%$ (patients 4 and 6) had a transition from hyperfibrinogenemia to hypofibrinogenemia. PCR was increased in $100 \%$ of cases, with a mean value of $38.5 \pm 15$, with values ranging from 6 to $96 \mathrm{mg} / \mathrm{L}$. Other changes found in the blood samples were: anemia $(85.71 \%)$, leukopenia $(42.85 \%)$, lymphopenia $(57.14 \%)$, thrombocytopenia $(42.85 \%)$ and thrombocytosis (28.57\%). Six patients (except patient 4 ) initially had a normal or low platelet count, followed by a tendency to increase towards the end of the disease. Liver damage manifested by hyperfermentemia was detected in 3 of the patients $(42.85 \%$ ), while renal impairment was present in $85.71 \%$ of cases.

All 7 cases required at least 24 hours in the intensive care unit, and 2 of them invasive ventilation (cases 4 and 6). All patients received individualized corticosteroid therapy and antibiotic therapy. Cases 1, 2, 5 and 7 were also given antiplatelet agents. Cases 4 and 6 required transfusions of freshly frozen plasma, albumin, forced diuresis and antiedema therapy. Five patients $(71.42 \%)$ had a positive therapeutic response, being discharged at home with recommendations to return later to follow-up the long-term evolution of the disease and the possible complications of coronavirus 2 . Unfortunately, cases 4 and 6 showed a refractory response to the treatment administered and died.

\section{Discussions}

The temporary association of MIS-C and the presence of serological evidence of SARS-CoV-2 infection in the seven children of study supports the pathogenic hypothesis that this syndrome is the result of a post-infectious immune response. Unlike the adult population, children with COVID19 who require respiratory ventilator support are fewer. According to specialized data, $57.14 \%$ of the children in the current study required mask oxygen therapy for at least 24 hours, and $28.57 \%$ required continuous oxygen therapy through the nasal cannula and even connected to the mechanical ventilation system. Patient 4 , aged 3 years, presented severe pulmonary damage manifested by severe bilateral inflammatory infiltration with preservation of subtotal pneumatization in S3-S5 on the right and S3, S6, S9 on the left and areas of early destruction. According to several studies, coronaviruses COVID19, SARS and MERS can induce the development of an excessive and ineffective host immune response. Pulmonary injury is much more severe in critically ill patients and is associated with the so-called "cytokine storm", characterized by increased plasma proinflammatory and anti-inflammatory cytokines.

Septic shock, MODS were the most common complications in critically ill patients. Multiorgan involvement in patients 4 and 6 included impairment of the nervous, hematopoietic and renal systems. Intravenous corticosteroid, antibiotic therapy and syndrome therapy were administered in accordance with the clinical situation of each patient separately. The pediatric patients mentioned in the study will require dynamic monitoring, taking into account the risk of heart damage and the unpredictable evolution of MIS-C.

\section{Conclusions}

Any child with a fever> 3 days during the COVID19 pandemic should be closely monitored and for the possibility of developing MIS-C and Kawasaki disease.

\section{References}

1. Castagnoli R, Votto M, Licari A, et al. (2020) Severe Acute Respiratory Syndrome Coronavirus 2 (SARS-CoV-2) Infection in Children and Adolescents: A Systematic Review. JAMA Pediatr. 174(9):882-889.

2. WHO. WHO coronavirus disease (COVID-19) dashboard. 2020 .

3. WHO. Multisystem inflammatory syndrome in children and adolescents with COVID-19.

4. European Centre for Disease Prevention and Control. Rapid risk assessment: paediatric inflammatory multisystem syndrome and SARS-CoV-2 infection in children. 2020.

5. Hennon, Teresa et al. (2020) COVID-19 associated Multisystem Inflammatory Syndrome in Children (MIS-C) guidelines; a Western New York approach. Progress in Pediatric Cardiology. 57. 101232.

6. European Centre for Disease Prevention and Control Rapid Risk Assessment: Paediatric inflammatory multisystem syndrome and SARS CoV 2 infection in children.

7. Dufort EM, Koumans EH, Chow EJ, et al. (2020) Multisystem Inflammatory Syndrome in Children in New York State. N Engl J Med. 383:347.

8. Li Jiang et.al. (2020) COVID-19 and multisystem inflammatory syndrome in children and adolescents. Lancet Infect Dis. Aug 17.

9. Kim GB, Park S, Kwon BS, Han JW, Park YW, Hong YM. (2014) Evaluation of the temporal association between Kawasaki disease and viral infections in South Korea. Korean Circ J. 44: 250-254.

10. Einat Blumfield and Terry L. Levin. (2020) COVID-19 in pediatric patients: a case series from the Bronx, NY; Pediatr Radiol. Jul 29: 1-6.

11. Sun, D., Li, H., Lu, X. et al. (2020) Clinical features of severe pediatric patients with coronavirus disease 2019 in Wuhan: a single center's observational study. World J Pediatr 16, 251259.

12. Cojocaru V, Ceban E, Irina Vasilieva, et al. (2020) Successful Resuscitation of Deadly SARS - Cov-2 / COVID19. Bioinformatics \& Proteomics Open Access Journal. 4:1:000134.

13. Vasilieva M, Vasilieva I, Vasiliev I, et al. (2019) Electro-Ion Membrane Distress Syndrome induces Chronic Fatigue Syndrome/Myalgic Encephalomyelitis (CFS/ME). Journal of Clinical Research in Anesthesiology. 2:2:1-6.

14. Irina V, Maria V, Vasiliev I, et al. (2019) Role of pCO2 (AV gap) of Multi Organ Dysfunction Syndrome. Journal of Biomedical and Pharmaceutical Sciences. 
15. Vasiliev I, Vasilieva M, Vasilieva I, et al. (2015) The extracorporeal bio-xeno perfuzion (myelo-timo-spleen) in multi-organ suportive therapy (MOST) as a modulator of energy, for immuno corection compensatory anti-inflamatory respons sindromes, and persistent inflammation, imunno supression, catabolism, and multi organ dysfunction. J Anesth Patient Care; 1:1045.
16. Vasiliev I, Vasilieva M, Vasilieva I, et al. (2019) The recruitment of microcirculatory-mitochondrial of critical obstetric situations in the complex multi-organ support therapy reduces pCO2 (AV gap) and the development of the syndrome of acute MODs. Biochem Mol Biol J.
This work is licensed under Creative

Commons Attribution 4.0 License

\section{To Submit Your Article Click Here: Submit Manuscript}

DOI: $10.31579 / 2692-9406 / 027$
Ready to submit your research? Choose Auctores and benefit from:

* fast, convenient online submission

* rigorous peer review by experienced research in your field

* rapid publication on acceptance

* authors retain copyrights

* unique DOI for all articles

* immediate, unrestricted online access

At Auctores, research is always in progress.

Learn more www.auctoresonline.org/journals/biomedical-research-andclinical-reviews- 